\title{
New Family of Multivalent Analytic Functions Defined on Complex Hilbert Space
}

\author{
Abbas Kareem Wanas ${ }^{1}$ and S. R. Swamy ${ }^{2}$ \\ ${ }^{1}$ Department of Mathematics, College of Science, University of Al-Qadisiyah, Diwaniyah, Iraq \\ e-mail: abbas.kareem.w@qu.edu.iq \\ ${ }^{2}$ Department of Computer Science and Engineering, R. V. College of Engineering, \\ Mysore Road, Bangalore-560 059, Karnataka, India \\ e-mail: swamysr@rvce.edu.in
}

\begin{abstract}
In this article, we define a certain new class of multivalent analytic functions with negative coefficients on complex Hilbert space. We derive a number of important geometric properties, such as, coefficient estimates, radii of starlikeness and convexity, extreme points and convex set.
\end{abstract}

\section{Introduction}

Let $\mathcal{A}_{p}$ indicate the family of functions $f$ of the form

$$
f(z)=z^{p}+\sum_{n=1}^{\infty} a_{n+p} z^{n+p} \quad(p \in \mathbb{N}=\{1,2, \ldots\}),
$$

which are analytic and multivalent in the open unit disk $U=\{z \in \mathbb{C}:|z|<1\}$.

Also, let $S_{p}$ denote the subclass of $\mathcal{A}_{p}$ consisting of functions of the form

Received: October 23, 2019; Accepted: January 20, 2020

2010 Mathematics Subject Classification: 30C45.

Keywords and phrases: multivalent function, Hilbert space, extreme point, convex set, radii of starlikeness and convexity.

Copyright (C) 2020 Abbas Kareem Wanas and S. R. Swamy. This is an open access article distributed under the Creative Commons Attribution License, which permits unrestricted use, distribution, and reproduction in any medium, provided the original work is properly cited. 


$$
f(z)=z^{p}-\sum_{n=1}^{\infty} a_{n+p} z^{n+p} \quad\left(a_{n+p} \geq 0, p \in \mathbb{N}=\{1,2, \ldots\}\right) .
$$

Denote by $H$ the Hilbert space on the complex field and $T$ by a linear operator on $H$. For a complex analytic function $f$ on the open unit disk $U$. The operator on $H$, indicated by $f(T)$, is defined by the well-known Riesz-Dunford integral [2].

$$
f(T)=\frac{1}{2 \pi i} \int_{c} f(z)(z I-T)^{-1} d z
$$

where $I$ is the identity operator on $H, c$ is a positively oriented simple closed rectifiable contour lying in $U$ and containing the spectrum $\sigma(T)$ of $T$ in its interior domain [3]. Also $f(T)$ can be defined by the series

$$
f(T)=\sum_{n=0}^{\infty} \frac{f^{(n)}(0)}{n !} T^{n},
$$

which converges in the norm topology [4].

Now, we define the class $W S_{p}(\delta, \alpha, \beta, T)$ consisting of the functions $f \in S_{p}$ such that

$$
\left\|\frac{2 \delta\left(\alpha^{p}-\beta^{p}\right) T f^{\prime}(T)}{f(\alpha T)-f(\beta T)}-p\right\|<p,
$$

where $0<\delta<1, \quad-1 \leq \beta<\alpha \leq 1, \quad \alpha \neq 0, \quad \beta \neq 0, \quad p \in \mathbb{N}$ and for all operator $T$ with $\|T\|<1, T \neq \varnothing(\varnothing$ denote the zero operator on $H)$.

The operator on Hilbert space were considered recently by Yu [8], Joshi [6], Kim et al. [1], Ghanim and Darus [5], Selvaraj et al. [7] and Wanas and Frasin [9].

\section{Main Results}

Theorem 2.1. Let $f \in S_{p}$ be given by (1.2). Then $f \in W S_{p}(\delta, \alpha, \beta, T)$ for all $T \neq \varnothing$ if and only if

$$
\sum_{n=1}^{\infty}\left[\delta(n+p)\left(\alpha^{p}-\beta^{p}\right)+p\left(\alpha^{n+p}-\beta^{n+p}\right)\right] a_{n+p}
$$




$$
\leq \frac{p}{2}\left(\alpha^{p}-\beta^{p}\right)(1-\|2 \delta-1\|)
$$

where $0<\delta<1,-1 \leq \beta<\alpha \leq 1, \alpha \neq 0, \beta \neq 0, p \in \mathbb{N}$.

The result is sharp for the function given by

$$
f(z)=z^{p}-\frac{p\left(\alpha^{p}-\beta^{p}\right)(1-\|2 \delta-1\|)}{2\left[\delta(n+p)\left(\alpha^{p}-\beta^{p}\right)+p\left(\alpha^{n+p}-\beta^{n+p}\right)\right]} z^{n+p}, \quad n \geq 1 .
$$

Proof. Let the inequality (2.1) holds. It is enough to show that

$$
\left\|2 \delta\left(\alpha^{p}-\beta^{p}\right) T f^{\prime}(T)-p(f(\alpha T)-f(\beta T))\right\|<p\|f(\alpha T)-f(\beta T)\| .
$$

We consider

$$
\begin{aligned}
& \left\|2 \delta\left(\alpha^{p}-\beta^{p}\right) T f^{\prime}(T)-p(f(\alpha T)-f(\beta T))\right\|-p\|f(\alpha T)-f(\beta T)\| \\
=\| p(2 \delta-1)\left(\alpha^{p}-\beta^{p}\right) T^{p} & \\
& \quad-\sum_{n=1}^{\infty}\left[2 \delta(n+p)\left(\alpha^{p}-\beta^{p}\right)-p\left(\alpha^{n+p}-\beta^{n+p}\right)\right] a_{n+p} T^{n+p} \| \\
& -p\left\|\left(\alpha^{p}-\beta^{p}\right) T^{p}-\sum_{n=1}^{\infty}\left(\alpha^{n+p}-\beta^{n+p}\right) a_{n+p} T^{n+p}\right\| \\
\leq & p\|2 \delta-1\|\left(\alpha^{p}-\beta^{p}\right)\|T\|^{p} \| \\
& +\sum_{n=1}^{\infty}\left[2 \delta(n+p)\left(\alpha^{p}-\beta^{p}\right)+p\left(\alpha^{n+p}-\beta^{n+p}\right)\right] a_{n+p}\|T\|^{n+p} \\
& -p\left(\alpha^{p}-\beta^{p}\right)\|T\|^{p}+p \sum_{n=1}^{\infty}\left(\alpha^{n+p}-\beta^{n+p}\right) a_{n+p}\|T\|^{n+p} \\
\leq & \sum_{n=1}^{\infty} 2\left[\delta(n+p)\left(\alpha^{p}-\beta^{p}\right)+p\left(\alpha^{n+p}-\beta^{n+p}\right)\right] a_{n+p}
\end{aligned}
$$




$$
-p\left(\alpha^{p}-\beta^{p}\right)(1-\|2 \delta-1\|) \leq 0 .
$$

Therefore, $f \in W S_{p}(\delta, \alpha, \beta, T)$.

To show the converse, let $f \in W S_{p}(\delta, \alpha, \beta, T)$. Then

$$
\left\|2 \delta\left(\alpha^{p}-\beta^{p}\right) T f^{\prime}(T)-p(f(\alpha T)-f(\beta T))\right\|<p\|f(\alpha T)-f(\beta T)\|,
$$

gives

$$
\begin{aligned}
& \left\|p(2 \delta-1)\left(\alpha^{p}-\beta^{p}\right) T^{p}-\sum_{n=1}^{\infty}\left[2 \delta(n+p)\left(\alpha^{p}-\beta^{p}\right)-p\left(\alpha^{n+p}-\beta^{n+p}\right)\right] a_{n+p} T^{n+p}\right\| \\
& <p\left\|\left(\alpha^{p}-\beta^{p}\right) T^{p}-\sum_{n=1}^{\infty}\left(\alpha^{n+p}-\beta^{n+p}\right) a_{n+p} T^{n+p}\right\| .
\end{aligned}
$$

Setting $T=r I(0<r<1)$ in the above inequality, we have

$$
\frac{p|2 \delta-1|\left(\alpha^{p}-\beta^{p}\right) r^{p}+\sum_{n=1}^{\infty}\left[2 \delta(n+p)\left(\alpha^{p}-\beta^{p}\right)+p\left(\alpha^{n+p}-\beta^{n+p}\right)\right] a_{n+p} r^{n+p}}{\left(\alpha^{p}-\beta^{p}\right) r^{p}-\sum_{n=1}^{\infty}\left(\alpha^{n+p}-\beta^{n+p}\right) a_{n+p} r^{n+p}}<p .
$$

Upon clearing denominator in (2.3) and letting $r \rightarrow 1$, we obtain

$$
\begin{aligned}
& p|2 \delta-1|\left(\alpha^{p}-\beta^{p}\right)+\sum_{n=1}^{\infty}\left[2 \delta(n+p)\left(\alpha^{p}-\beta^{p}\right)+p\left(\alpha^{n+p}-\beta^{n+p}\right)\right] a_{n+p} \\
& <p\left(\alpha^{p}-\beta^{p}\right) r^{p}-p \sum_{n=1}^{\infty}\left(\alpha^{n+p}-\beta^{n+p}\right) a_{n+p} .
\end{aligned}
$$

Thus

$$
\sum_{n=1}^{\infty}\left[\delta(n+p)\left(\alpha^{p}-\beta^{p}\right)+p\left(\alpha^{n+p}-\beta^{n+p}\right)\right] a_{n+p} \leq \frac{p}{2}\left(\alpha^{p}-\beta^{p}\right)(1-\|2 \delta-1\|),
$$

which completes the proof. 
Corollary 2.1. If $f \in W S_{p}(\delta, \alpha, \beta, T)$, then

$$
a_{n+p} \leq \frac{p\left(\alpha^{p}-\beta^{p}\right)(1-\|2 \delta-1\|)}{2\left[\delta(n+p)\left(\alpha^{p}-\beta^{p}\right)+p\left(\alpha^{n+p}-\beta^{n+p}\right)\right]}, \quad n \geq 1 .
$$

Theorem 2.2. If $f \in W S_{p}(\delta, \alpha, \beta, T)$ and $\|T\|<1, T \neq \varnothing$, then

$$
\begin{aligned}
& \|T\|^{p}-\frac{p\left(\alpha^{p}-\beta^{p}\right)(1-\|2 \delta-1\|)}{2\left[\delta(p+1)\left(\alpha^{p}-\beta^{p}\right)+p\left(\alpha^{p+1}-\beta^{p+1}\right)\right]}\|T\|^{p+1} \leq\|f(T)\| \\
\leq & \|T\|^{p}+\frac{p\left(\alpha^{p}-\beta^{p}\right)(1-\|2 \delta-1\|)}{2\left[\delta(p+1)\left(\alpha^{p}-\beta^{p}\right)+p\left(\alpha^{p+1}-\beta^{p+1}\right)\right]}\|T\|^{p+1}
\end{aligned}
$$

and

$$
\begin{aligned}
& p\|T\|^{p-1}-\frac{p(n+p)\left(\alpha^{p}-\beta^{p}\right)(1-\|2 \delta-1\|)}{2\left[\delta(n+p)\left(\alpha^{p}-\beta^{p}\right)+p\left(\alpha^{n+p}-\beta^{n+p}\right)\right]}\|T\|^{p} \leq\left\|f^{\prime}(T)\right\| \\
\leq & p\|T\|^{p-1}+\frac{p(n+p)\left(\alpha^{p}-\beta^{p}\right)(1-\|2 \delta-1\|)}{2\left[\delta(n+p)\left(\alpha^{p}-\beta^{p}\right)+p\left(\alpha^{n+p}-\beta^{n+p}\right)\right]}\|T\|^{p} .
\end{aligned}
$$

Proof. According to Theorem 2.1, we have

$$
\sum_{n=1}^{\infty} a_{n+p} \leq \frac{p\left(\alpha^{p}-\beta^{p}\right)(1-\|2 \delta-1\|)}{2\left[\delta(p+1)\left(\alpha^{p}-\beta^{p}\right)+p\left(\alpha^{p+1}-\beta^{p+1}\right)\right]} .
$$

Thus

$$
\begin{aligned}
\|f(T)\| & \geq\|T\|^{p}-\sum_{n=1}^{\infty} a_{n+p}\|T\|^{n+p} \geq\|T\|^{p}-\|T\|^{p+1}-\sum_{n=1}^{\infty} a_{n+p} \\
& \geq\|T\|^{p}-\frac{p\left(\alpha^{p}-\beta^{p}\right)(1-\|2 \delta-1\|)}{2\left[\delta(p+1)\left(\alpha^{p}-\beta^{p}\right)+p\left(\alpha^{p+1}-\beta^{p+1}\right)\right]}\|T\|^{p+1} .
\end{aligned}
$$

Also,

$$
\|f(T)\| \leq\|T\|^{p}+\sum_{n=1}^{\infty} a_{n+p}\|T\|^{n+p}
$$




$$
\leq\|T\|^{p}+\frac{p\left(\alpha^{p}-\beta^{p}\right)(1-\|2 \delta-1\|)}{2\left[\delta(p+1)\left(\alpha^{p}-\beta^{p}\right)+p\left(\alpha^{p+1}-\beta^{p+1}\right)\right]}\|T\|^{p+1}
$$

In the light of Theorem 2.1, we obtain

$$
\sum_{n=1}^{\infty}(n+p) a_{n+p} \leq \frac{p(n+p)\left(\alpha^{p}-\beta^{p}\right)(1-\|2 \delta-1\|)}{2\left[\delta(n+p)\left(\alpha^{p}-\beta^{p}\right)+p\left(\alpha^{n+p}-\beta^{n+p}\right)\right]} .
$$

Hence

$$
\begin{aligned}
\left\|f^{\prime}(T)\right\| & \geq p\|T\|^{p-1}-\sum_{n=1}^{\infty}(n+p) a_{n+p}\|T\|^{n+p-1} \\
& \geq p\|T\|^{p-1}-\|T\|^{p} \sum_{n=1}^{\infty}(n+p) a_{n+p} \\
& \geq p\|T\|^{p-1}-\frac{p(n+p)\left(\alpha^{p}-\beta^{p}\right)(1-\|2 \delta-1\|)}{2\left[\delta(n+p)\left(\alpha^{p}-\beta^{p}\right)+p\left(\alpha^{n+p}-\beta^{n+p}\right)\right]}\|T\|^{p}
\end{aligned}
$$

and

$$
\begin{aligned}
\left\|f^{\prime}(T)\right\| & \leq p\|T\|^{p-1}+\|T\|^{p} \sum_{n=1}^{\infty}(n+p) a_{n+p} \\
& \leq p\|T\|^{p-1}+\frac{p(n+p)\left(\alpha^{p}-\beta^{p}\right)(1-\|2 \delta-1\|)}{2\left[\delta(n+p)\left(\alpha^{p}-\beta^{p}\right)+p\left(\alpha^{n+p}-\beta^{n+p}\right)\right]}\|T\|^{p} .
\end{aligned}
$$

Therefore the proof is complete.

Theorem 2.3. If $f \in W S_{p}(\delta, \alpha, \beta, T)$, then $f$ will be multivalent starlike of order $\theta(0 \leq \theta<p)$ in the disk $|z|<r_{1}$, where

$$
r_{1}=\inf _{n}\left\{\frac{2(p-\theta)\left[\delta(n+p)\left(\alpha^{p}-\beta^{p}\right)+p\left(\alpha^{n+p}-\beta^{n+p}\right)\right]}{p(n+p-\theta)\left(\alpha^{p}-\beta^{p}\right)(1-\|2 \delta-1\|)}\right\}^{\frac{1}{n}}, \quad(n \geq 1) .
$$

The result is sharp for the function $f$ given by (2.2). 
Proof. It is sufficient to show that

$$
\left\|\frac{T f^{\prime}(T)}{f(T)}-p\right\| \leq p-\theta
$$

We get

$$
\left\|\frac{T f^{\prime}(T)}{f(T)}-p\right\| \leq \frac{\sum_{n=1}^{\infty} n a_{n+p}\|T\|^{n}}{1-\sum_{n=1}^{\infty} a_{n+p}\|T\|^{n}} .
$$

Hence (2.4) will be satisfied if

$$
\sum_{n=1}^{\infty}\left(\frac{n+p-\theta}{p-\theta}\right) a_{n+p}\|T\|^{n} \leq 1 .
$$

In the light of Theorem 2.1, if $f \in W S_{p}(\delta, \alpha, \beta, T)$, then

$$
\sum_{n=1}^{\infty} \frac{2\left[\delta(n+p)\left(\alpha^{p}-\beta^{p}\right)+p\left(\alpha^{n+p}-\beta^{n+p}\right)\right]}{p\left(\alpha^{p}-\beta^{p}\right)(1-\|2 \delta-1\|)} a_{n+p} \leq 1
$$

By making use of (2.6), we see that (2.5) holds true if

$$
\frac{n+p-\theta}{p-\theta}\|T\|^{n} \leq \frac{2\left[\delta(n+p)\left(\alpha^{p}-\beta^{p}\right)+p\left(\alpha^{n+p}-\beta^{n+p}\right)\right]}{p\left(\alpha^{p}-\beta^{p}\right)(1-\|2 \delta-1\|)}
$$

or equivalently

$$
\|T\| \leq\left\{\frac{2(p-\theta)\left[\delta(n+p)\left(\alpha^{p}-\beta^{p}\right)+p\left(\alpha^{n+p}-\beta^{n+p}\right)\right]}{p(n+p-\theta)\left(\alpha^{p}-\beta^{p}\right)(1-\|2 \delta-1\|)}\right\}^{\frac{1}{n}} .
$$

This gives the desired result.

Theorem 2.4. If $f \in W S_{p}(\delta, \alpha, \beta, T)$, then $f$ is multivalent convex of order $\theta(0 \leq \theta<p)$ in the disk $|z|<r_{2}$, where 


$$
r_{2}=\inf _{n}\left\{\frac{2(p-\theta)\left[\delta(n+p)\left(\alpha^{p}-\beta^{p}\right)+p\left(\alpha^{n+p}-\beta^{n+p}\right)\right]}{(n+p)(n+p-\theta)\left(\alpha^{p}-\beta^{p}\right)(1-\|2 \delta-1\|)}\right\}^{\frac{1}{n}},(n \geq 1)
$$

The result is sharp for the function $f$ given by (2.2).

Proof. It is sufficient to show that

$$
\left\|\frac{T f^{\prime \prime}(T)}{f^{\prime}(T)}+1-p\right\| \leq p-\theta
$$

The result follows by application of arguments similar to the proof of Theorem 2.3.

Theorem 2.5. Let $f_{0}(z)=z^{p}$ and

$$
f_{n}(z)=z^{p}-\frac{p\left(\alpha^{p}-\beta^{p}\right)(1-\|2 \delta-1\|)}{2\left[\delta(n+p)\left(\alpha^{p}-\beta^{p}\right)+p\left(\alpha^{n+p}-\beta^{n+p}\right)\right]} z^{n+p}, n \geq 1 .
$$

Then $f \in W S_{p}(\delta, \alpha, \beta, T)$ if and only if it can be expressed in the form:

$$
f(z)=\sum_{n=0}^{\infty} \lambda_{n} f_{n}(z)
$$

where $\lambda_{n} \geq 0$ and $\sum_{n=0}^{\infty} \lambda_{n}=1$.

Proof. Assume that $f$ can be expressed by (2.7). Then, we find that

$$
\begin{aligned}
f(z) & =\sum_{n=0}^{\infty} \lambda_{n} f_{n}(z)=\lambda_{0} f_{0}(z)+\sum_{n=0}^{\infty} \lambda_{n} f_{n}(z) \\
& =z^{p}-\sum_{n=1}^{\infty} \frac{p\left(\alpha^{p}-\beta^{p}\right)(1-\|2 \delta-1\|)}{2\left[\delta(n+p)\left(\alpha^{p}-\beta^{p}\right)+p\left(\alpha^{n+p}-\beta^{n+p}\right)\right]} \lambda_{n} z^{n+p} .
\end{aligned}
$$

Thus

$$
\begin{aligned}
& \sum_{n=1}^{\infty} \frac{2\left[\delta(n+p)\left(\alpha^{p}-\beta^{p}\right)+p\left(\alpha^{n+p}-\beta^{n+p}\right)\right]}{p\left(\alpha^{p}-\beta^{p}\right)(1-\|2 \delta-1\|)} \\
& \quad \times \frac{p\left(\alpha^{p}-\beta^{p}\right)(1-\|2 \delta-1\|)}{2\left[\delta(n+p)\left(\alpha^{p}-\beta^{p}\right)+p\left(\alpha^{n+p}-\beta^{n+p}\right)\right]} \lambda_{n}
\end{aligned}
$$




$$
=\sum_{n=1}^{\infty} \lambda_{n}=1-\lambda_{0} \leq 1
$$

and so $f \in W S_{p}(\delta, \alpha, \beta, T)$.

Conversely, suppose that $f$ given by (1.2) is in the class $W S_{p}(\delta, \alpha, \beta, T)$. Then by Corollary 2.1, we have

$$
a_{n+p} \leq \frac{p\left(\alpha^{p}-\beta^{p}\right)(1-\|2 \delta-1\|)}{2\left[\delta(n+p)\left(\alpha^{p}-\beta^{p}\right)+p\left(\alpha^{n+p}-\beta^{n+p}\right)\right]} .
$$

Putting

$$
\lambda_{n}=\frac{2\left[\delta(n+p)\left(\alpha^{p}-\beta^{p}\right)+p\left(\alpha^{n+p}-\beta^{n+p}\right)\right]}{p\left(\alpha^{p}-\beta^{p}\right)(1-\|2 \delta-1\|)} a_{n}, \quad n \geq 1,
$$

and $\lambda_{0}=1-\sum_{n=1}^{\infty} \lambda_{n}$. Then

$$
\begin{aligned}
f(z) & =z^{p}-\sum_{n=1}^{\infty} a_{n+p} z^{n+p} \\
& =z^{p}-\sum_{n=1}^{\infty} \frac{p\left(\alpha^{p}-\beta^{p}\right)(1-\|2 \delta-1\|)}{2\left[\delta(n+p)\left(\alpha^{p}-\beta^{p}\right)+p\left(\alpha^{n+p}-\beta^{n+p}\right)\right]} \lambda_{n} z^{n+p} \\
& =z^{p}-\sum_{n=1}^{\infty}\left(z^{p}-f_{n}(z)\right) \lambda_{n}=\left(1-\sum_{n=1}^{\infty} \lambda_{n}\right) z^{p}+\sum_{n=1}^{\infty} \lambda_{n} f_{n}(z) \\
& =\lambda_{0} f_{0}(z)+\sum_{n=1}^{\infty} \lambda_{n} f_{n}(z)=\sum_{n=0}^{\infty} \lambda_{n} f_{n}(z),
\end{aligned}
$$

which completes the proof.

Theorem 2.6. The class $W S_{p}(\delta, \alpha, \beta, T)$ is a convex set.

Proof. Let $f_{1}$ and $f_{2}$ be the arbitrary elements of $W S_{p}(\delta, \alpha, \beta, T)$. Then for every $t(0 \leq t \leq 1)$, we show that $(1-t) f_{1}+t f_{2} \in W S_{p}(\delta, \alpha, \beta, T)$. Thus, we obtain 


$$
(1-t) f_{1}+t f_{2}=z^{p}-\sum_{n=1}^{\infty}\left((1-t) a_{n+p}+t b_{n+p}\right) z^{n+p}
$$

Hence

$$
\begin{aligned}
& \sum_{n=1}^{\infty}\left[\delta(n+p)\left(\alpha^{p}-\beta^{p}\right)+p\left(\alpha^{n+p}-\beta^{n+p}\right)\right]\left((1-t) a_{n+p}+t b_{n+p}\right) \\
= & (1-t) \sum_{n=1}^{\infty}\left[\delta(n+p)\left(\alpha^{p}-\beta^{p}\right)+p\left(\alpha^{n+p}-\beta^{n+p}\right)\right] a_{n+p} \\
& +t \sum_{n=1}^{\infty}\left[\delta(n+p)\left(\alpha^{p}-\beta^{p}\right)+p\left(\alpha^{n+p}-\beta^{n+p}\right)\right] b_{n+p} \\
\leq & \frac{p}{2}(1-t)\left(\alpha^{p}-\beta^{p}\right)(1-\|2 \delta-1\|)+\frac{p}{2} t\left(\alpha^{p}-\beta^{p}\right)(1-\|2 \delta-1\|) \\
= & \frac{p}{2}\left(\alpha^{p}-\beta^{p}\right)(1-\|2 \delta-1\|) .
\end{aligned}
$$

This completes the proof.

\section{References}

[1] Y. C. Kim, J. S. Lee and S. H. Lee, A certain subclass of analytic functions with negative coefficients for operators on Hilbert space, Math. Japon. 47(1) (1998), 115-124.

[2] N. Dunford and J. T. Schwartz, Linear Operators, Part I, General Theory, New YorkLondon: Interscience Publishers, 1958.

[3] K. Fan, Analytic functions of a proper contraction, Math. Z. 160 (1978), 275-290. https://doi.org/10.1007/BF01237041

[4] K. Fan, Julia's lemma for operators, Math. Ann. 239 (1979), 241-245. https://doi.org/10.1007/BF01351489

[5] F. Ghanim and M. Darus, On new subclass of analytic $p$-valent function with negative coefficients for operator on Hilbert space, Int. Math. Forum 3(2) (2008), 69-77.

[6] S. B. Joshi, On a class of analytic functions with negative coefficient for operators on Hilbert Space, J. Appr. Theory and Appl. (1998), 107-112. 
[7] C. Selvaraj, A. A. J. Pamela and M. Thirucheran, On a subclass of multivalent analytic functions with negative coefficients for contraction operators on Hilbert space, Int. J. Contemp. Math. Sci. 4(9) (2009), 447-456.

[8] Xiao Pei Yu, A subclass of analytic $p$-valent functions for operator on Hilbert Space, Math. Japon. 40(2) (1994), 303-308.

[9] A. K. Wanas and B. A. Frasin, Applications of fractional calculus for a certain subclass of multivalent analytic functions on complex Hilbert space, General Mathematics 26(1-2) (2018), 11-23. 